\title{
Asymptotic Method of Solution for a Problem of Construction of Optimal Gas-Lift Process Modes
}

\author{
Fikrat A. Aliev, ${ }^{1}$ Mutallim M. Mutallimov, ${ }^{1}$ Idrak M. Askerov, ${ }^{1}$ \\ and Iouldouz S. Raguimov ${ }^{2}$ \\ ${ }^{1}$ Institute of Applied Mathematics, Baku State University, Baku 1148, Azerbaijan \\ ${ }^{2}$ Department of Mathematics and Statistics, York University, Toronto, ON, Canada M3J 1P3 \\ Correspondence should be addressed to Fikrat A. Aliev, f_aliev@yahoo.com
}

Received 22 December 2009; Revised 16 April 2010; Accepted 16 April 2010

Academic Editor: Ben T. Nohara

Copyright (C) 2010 Fikrat A. Aliev et al. This is an open access article distributed under the Creative Commons Attribution License, which permits unrestricted use, distribution, and reproduction in any medium, provided the original work is properly cited.

\begin{abstract}
Mathematical model in oil extraction by gas-lift method for the case when the reciprocal value of well's depth represents a small parameter is considered. Problem of optimal mode construction (i.e., construction of optimal program trajectories and controls) is reduced to the linear-quadratic optimal control problem with a small parameter. Analytic formulae for determining the solutions at the first-order approximation with respect to the small parameter are obtained. Comparison of the obtained results with known ones on a specific example is provided, which makes it, in particular, possible to use obtained results in realizations of oil extraction problems by gas-lift method.
\end{abstract}

\section{Introduction}

It is known [1-3] that the gas-lift technique of exploitation of oil wells is widely used when the gushing method does not work for the reason of insufficiency in pressure. The essence of the gas-lift method consists of the fact that by the mean of energy of injected underground gas it is possible to lift fluid to the surface.

While the gas-lift method is widely used in oil extraction for a sufficiently long period of time, construction of an adequate mathematical model is rather an actual problem. Mathematical model describing the oil lifting process in pump-compressor tubes is described in [4].

In $[4,5]$, using this model, an optimal control problem for gas-lift process is formulated, where the pressure and volume of the injected gas is used as a control parameter. In the same papers, the method of straight lines is used to reduce the optimal control problem 
to the linear-quadratic optimal control problem (LQOCP). It should be noted that, when the depth of a well is large, in order to obtain sufficiently accurate results it is necessary to divide this distance into large number of relatively small segments. The latter, in its turn increases the dimensions of the system and, consequently, results in higher volume of computations to solve such problems. Thus, generally speaking, solution of these problems yields higher approximation and calculation errors.

Therefore, it seems quite rational to develop other solution methods with lower computational complicatedness [6-8]. One of the methods applicable to the considered problem for the case when the reciprocal value of well's depth represents a small parameter is the asymptotic method described in [9].

In the present paper, using the method of straight lines, the mathematical model of the gas-lift process is represented as a system of partial differential equations of hyperbolic type. In a particular case, we arrive to LQOCP for a system of ordinary differential equations containing a small parameter. Applying the algorithm introduced in [10,11], the solution to LQOCP is obtained as a function of the small parameter. Consequently, as in $[6,9]$, at the first approximation, analytic formulas for the volume of injected gas (control) and production level (trajectories) are obtained. As it supposed, the presented approach will reduce substantially the amount of required computations.

\section{Mathematical Formulation of the Problem}

As in [4], mathematical model of gas-fluid mixture flow in pipes is described by the system of hyperbolic-type partial differential equations:

$$
\begin{gathered}
\frac{\partial P}{\partial t}=-\frac{c^{2}}{\bar{F}} \frac{\partial Q}{\partial x} \\
\frac{\partial Q}{\partial t}=-\bar{F} \frac{\partial P}{\partial x}-2 a Q
\end{gathered} \quad t \geq 0, x \in[0,2 L]
$$

which for $x=z / 2 L$ and $\varepsilon=1 / 2 L$ can be written as

$$
\begin{gathered}
\frac{\partial P}{\partial t}=-\frac{c^{2}}{F} \frac{\partial Q}{\partial z} \varepsilon, \quad z \in(0,1) \\
\frac{\partial Q}{\partial t}=-F \frac{\partial P}{\partial z} \varepsilon-2 a Q
\end{gathered}
$$

with appropriate boundary and initial conditions

$$
\begin{gathered}
P(z, 0)=P^{0}(z), \quad Q(z, 0)=Q^{0}(z), \quad P(0, t)=P_{0}(t), \quad Q(0, t)=Q_{0}(t), \\
P(L+0, t)=P(L-0, t)+P_{p l}(t), \quad Q(L+0, t)=Q(L-0, t)+Q_{p l}(t) .
\end{gathered}
$$


It is required to determine a control

$$
U(t)=\left[P_{0}(t), Q_{0}(t)\right]^{\prime}
$$

minimizing the functional

$$
J=\frac{1}{2} \alpha\left(Q(2 L, T)-Q_{\mathrm{deb}}\right)^{2}+\frac{1}{2} \int_{0}^{T} U^{\prime}(t) R U(t) d t
$$

where $P$ is pressure, $Q$ is gas-fluid mixture volume and $Q_{\text {deb }}$ is the desired yield.

Applying the so-called method of straight lines to (2.2) and taking $l=1 / N$, we obtain

$$
\begin{gathered}
\frac{d P_{k}}{d t}=-\frac{c^{2} \varepsilon}{F \cdot l}\left(Q_{k}-Q_{k-1}\right), \\
\frac{d Q_{k}}{d t}=-\frac{F \varepsilon}{l}\left(P_{k}-P_{k-1}\right)-2 a Q_{k} .
\end{gathered}
$$

Note that for $k=N+1(2.6)$ can be written as

$$
\begin{gathered}
\dot{P}_{N+1}=-\frac{c_{2}^{2} \varepsilon}{F_{2} l} Q_{N+1}+\frac{c_{2}^{2} \varepsilon}{F_{2} l} Q_{N}+\frac{c_{2} \varepsilon}{F_{2} l} Q_{p l} \\
\dot{Q}_{N+1}=-\frac{F_{2} \varepsilon}{l} P_{N+1}+\frac{F_{2} \varepsilon}{l} P_{N}-2 a_{2} Q_{N+1}+\frac{F_{2} \varepsilon}{l} P_{p l},
\end{gathered}
$$

where $Q_{p l}, P_{p l}$ denote gas-fluid outlay (yield) and pressure at the bottom of a well, respectively. As in [10], a linear-quadratic optimal control problem is formulated for this system. It is required to find $x, u$ satisfying the equation

$$
\dot{x}=\left(A_{0}+A_{1} \varepsilon\right) x+B \varepsilon u+C \varepsilon
$$

with initial condition

$$
x(0)=x^{0}
$$

such that the value of the functional

$$
J=\frac{1}{2}(x(T)-\bar{x})^{\prime} K(x(T)-\bar{x})+\frac{1}{2} \int_{0}^{T} u^{\prime} R u d t
$$

is minimized. 
Here,

$$
\begin{aligned}
& A_{1}=\left[\begin{array}{cccccccccccccc}
A_{11} & 0 & 0 & 0 & \cdots & 0 & 0 & 0 & 0 & \cdots & 0 & 0 & 0 & 0 \\
A_{12} & A_{11} & 0 & 0 & \cdots & 0 & 0 & 0 & 0 & \cdots & 0 & 0 & 0 & 0 \\
0 & A_{12} & A_{11} & 0 & \cdots & 0 & 0 & 0 & 0 & \cdots & 0 & 0 & 0 & 0 \\
0 & 0 & A_{12} & A_{11} & \cdots & 0 & 0 & 0 & 0 & \cdots & 0 & 0 & 0 & 0 \\
\cdots & \cdots & \cdots & \cdots & \cdots & \cdots & \cdots & \cdots & \cdots & \cdots & \cdots & \cdots & \cdots & \cdots \\
0 & 0 & 0 & 0 & \cdots & A_{11} & 0 & 0 & 0 & \cdots & 0 & 0 & 0 & 0 \\
0 & 0 & 0 & 0 & \cdots & A_{12} & A_{11} & 0 & 0 & \cdots & 0 & 0 & 0 & 0 \\
0 & 0 & 0 & 0 & \cdots & 0 & A_{21} & A_{22} & 0 & \cdots & 0 & 0 & 0 & 0 \\
0 & 0 & 0 & 0 & \cdots & 0 & 0 & A_{21} & A_{22} & \cdots & 0 & 0 & 0 & 0 \\
\cdots & \cdots & \cdots & \cdots & \cdots & \cdots & \cdots & \cdots & \cdots & \cdots & \cdots & \cdots & \cdots & \cdots \\
0 & 0 & 0 & 0 & \cdots & 0 & 0 & 0 & 0 & \cdots & A_{22} & 0 & 0 & 0 \\
0 & 0 & 0 & 0 & \cdots & 0 & 0 & 0 & 0 & \cdots & A_{21} & A_{22} & 0 & 0 \\
0 & 0 & 0 & 0 & \cdots & 0 & 0 & 0 & 0 & \cdots & 0 & A_{21} & A_{22} & 0 \\
0 & 0 & 0 & 0 & \cdots & 0 & 0 & 0 & 0 & \cdots & 0 & 0 & A_{21} & A_{22}
\end{array}\right], \\
& A_{0}=\left[\begin{array}{cccccccccccccc}
0 & 0 & 0 & 0 & \cdots & 0 & 0 & 0 & 0 & \cdots & 0 & 0 & 0 & 0 \\
0 & -2 a_{1} & 0 & 0 & \cdots & 0 & 0 & 0 & 0 & \cdots & 0 & 0 & 0 & 0 \\
0 & 0 & 0 & 0 & \cdots & 0 & 0 & 0 & 0 & \cdots & 0 & 0 & 0 & 0 \\
0 & 0 & 0 & -2 a_{1} & \cdots & 0 & 0 & 0 & 0 & \cdots & 0 & 0 & 0 & 0 \\
\cdots & \cdots & \cdots & \cdots & \cdots & \cdots & \cdots & \cdots & \cdots & \cdots & \cdots & \cdots & \cdots & \cdots \\
0 & 0 & 0 & 0 & \cdots & 0 & 0 & 0 & 0 & \cdots & 0 & 0 & 0 & 0 \\
0 & 0 & 0 & 0 & \cdots & 0 & -2 a_{1} & 0 & 0 & \cdots & 0 & 0 & 0 & 0 \\
0 & 0 & 0 & 0 & \cdots & 0 & 0 & 0 & 0 & \cdots & 0 & 0 & 0 & 0 \\
0 & 0 & 0 & 0 & \cdots & 0 & 0 & 0 & -2 a_{2} & \cdots & 0 & 0 & 0 & 0 \\
\cdots & \cdots & \cdots & \cdots & \cdots & \cdots & \cdots & \cdots & \cdots & \cdots & \cdots & \cdots & \cdots & \cdots \\
0 & 0 & 0 & 0 & \cdots & 0 & 0 & 0 & 0 & \cdots & 0 & 0 & 0 & 0 \\
0 & 0 & 0 & 0 & \cdots & 0 & 0 & 0 & 0 & \cdots & 0 & -2 a_{2} & 0 & 0 \\
0 & 0 & 0 & 0 & \cdots & 0 & 0 & 0 & 0 & \cdots & 0 & 0 & 0 & 0 \\
0 & 0 & 0 & 0 & \cdots & 0 & 0 & 0 & 0 & \cdots & 0 & 0 & 0 & -2 a_{2}
\end{array}\right], \\
& A_{11}=\left[\begin{array}{cc}
0 & -\frac{c_{1}^{2}}{F_{1} l} \\
-\frac{F_{1}}{l} & 0
\end{array}\right], \quad A_{12}=\left[\begin{array}{cc}
0 & \frac{c_{1}^{2}}{F_{1} l} \\
\frac{F_{1}}{l} & 0
\end{array}\right], \quad A_{22}=\left[\begin{array}{cc}
0 & -\frac{c_{2}^{2}}{F_{2} l} \\
-\frac{F_{2}}{l} & 0
\end{array}\right], \quad A_{21}=\left[\begin{array}{cc}
0 & \frac{c_{2}^{2}}{F_{2} l} \\
\frac{F_{2}}{l} & 0
\end{array}\right] \text {, } \\
& x=\left[P_{1}, Q_{1}, P_{2}, Q_{2}, \ldots, P_{N}, Q_{N}, \ldots, P_{2 N}, Q_{2 N}\right]^{\prime} \text {, } \\
& x_{0}=\left[P_{1}^{0}, Q_{1}^{0}, P_{2}^{0}, Q_{2}^{0}, \ldots, P_{N}^{0}, Q_{N}^{0}, \ldots, P_{2 N}^{0}, Q_{2 N}^{0}\right]^{\prime},
\end{aligned}
$$




$$
\begin{gathered}
u=\left[\begin{array}{l}
P_{0} \\
Q_{0}
\end{array}\right], \\
B=\left[\begin{array}{cc}
0 & \frac{c_{1}^{2}}{F_{1} l} \\
\frac{F_{1}}{l} & 0 \\
0 & 0 \\
\cdots & \cdots \\
\cdots & \cdots \\
0 & 0 \\
0 & 0 \\
0 & 0
\end{array}\right], \quad C=\left[\begin{array}{cc}
0 & 0 \\
\ldots & \cdots \\
0 & 0 \\
0 & \frac{c_{2}^{2}}{F_{2} l} \\
\frac{F_{2}}{l} & 0 \\
0 & 0 \\
\cdots & \cdots \\
0 & 0
\end{array}\right]\left[\begin{array}{l}
P_{p l} \\
Q_{p l}
\end{array}\right] .
\end{gathered}
$$

Let $R=\bar{R} \varepsilon$. Then the corresponding Euler-Lagrange control problem can be written as

$$
\begin{gathered}
{\left[\begin{array}{c}
\dot{x} \\
\dot{\lambda}
\end{array}\right]=\left[\begin{array}{cc}
A_{0}+A_{1} \varepsilon & -\varepsilon B \bar{R}^{-1} B^{\prime} \\
0 & -A_{0}^{1}-A_{1}^{1} \varepsilon
\end{array}\right]\left[\begin{array}{l}
x \\
\lambda
\end{array}\right]+\left[\begin{array}{c}
C \varepsilon \\
0
\end{array}\right],} \\
x(0)=x_{0} \\
\lambda(T)=N(x(T)-\bar{x}) .
\end{gathered}
$$

Thus, initial problem (2.2)-(2.5) is reduced to finding the solution of problem (2.12), where $\varepsilon$ is a small parameter. Therefore, the asymptotic method (see [9]) allowing to expand the solution of system (2.12) with respect to small parameter $\varepsilon$ can be applied.

\section{Application of Asymptotic Method}

Let us apply the asymptotic method to the Euler-Lagrange equation (2.12) with boundary conditions for $x(0)$ and $\lambda(T)$. According to [12],

$$
\begin{aligned}
{\left[\begin{array}{l}
x(T) \\
\lambda(T)
\end{array}\right]=} & e^{\left[\begin{array}{cc}
A_{0}+\varepsilon A_{1}-\varepsilon B \bar{R}^{-1} B^{\prime} \\
0 & -A_{0}^{\prime}-\varepsilon A_{1}^{\prime}
\end{array}\right]^{T}\left[\begin{array}{c}
x_{0} \\
\lambda(0)
\end{array}\right]} \\
& +\left[e^{\left[\begin{array}{cc}
A_{0}+\varepsilon A_{1}-\varepsilon B \bar{R}^{-1} B^{\prime} \\
0 & -A_{0}^{\prime}-\varepsilon A_{1}^{\prime}
\end{array}\right]^{T}}-\left[\begin{array}{cc}
E & 0 \\
0 & E
\end{array}\right]\right]\left[\begin{array}{cc}
A_{0}+\varepsilon A_{1} & -\varepsilon B \bar{R}^{-1} B^{\prime} \\
0 & -A_{0}^{\prime}-\varepsilon A_{1}^{\prime}
\end{array}\right]^{-1}\left[\begin{array}{c}
C \varepsilon \\
0
\end{array}\right] .
\end{aligned}
$$

Further, let us expand the expression

$$
e^{\left[\begin{array}{cc}
A_{0}+\varepsilon A_{1} & -\varepsilon B \bar{R}^{-1} B^{\prime} \\
0 & -A_{0}^{\prime}-\varepsilon A_{1}^{\prime}
\end{array}\right] T}=e^{\left[\begin{array}{cc}
A_{0} & 0 \\
0 & -A_{0}^{\prime}
\end{array}\right] T+\varepsilon\left[\begin{array}{cc}
A_{1} & -B \bar{R}^{-1} B^{\prime} \\
0 & -A_{1}^{\prime}
\end{array}\right] T}
$$


from (3.1) with respect to $\varepsilon$. If we denote

$$
H_{1} \stackrel{\text { i.e }}{=}\left[\begin{array}{cc}
A_{0} & 0 \\
0 & -A_{0}^{\prime}
\end{array}\right], \quad H_{2} \stackrel{\text { i.e }}{=}\left[\begin{array}{cc}
A_{1} & -B \bar{R}^{-1} B^{\prime} \\
0 & -A_{1}^{\prime}
\end{array}\right] \text {, }
$$

then according to [12], the expansion of expression (3.2) with respect to $\varepsilon$ can be represented as:

$$
e^{H_{1} T+\varepsilon H_{2} T} \approx e^{H_{1} T}+\varepsilon \int_{0}^{1} e^{H_{1} T(1-s)} H_{2} T e^{H_{1} T s} d s
$$

Denote the integral in (3.4) by $L_{0}$. Then it is not difficult to show that matrix $L_{0}$ is a solution of the following Sylvester's equation:

$$
H_{1} L_{0}-L_{0} H_{1}=e^{H_{1} T} H_{2}-H_{2} e^{H_{1} T} .
$$

Therefore, for expression (3.2) we obtain the expansion

$$
e^{H_{1} T+\varepsilon H_{2} T} \approx e^{H_{1} T}+\varepsilon L_{0} .
$$

Introducing notations

$$
L_{0}=\left[\begin{array}{ll}
L_{1} & L_{2} \\
L_{3} & L_{4}
\end{array}\right], \quad\left[\begin{array}{cc}
A_{0}+\varepsilon A_{1} & -\varepsilon B \bar{R}^{-1} B^{\prime} \\
0 & -A_{0}^{\prime}-\varepsilon A_{1}^{\prime}
\end{array}\right]^{-1}=\left[\begin{array}{cc}
S_{1} & S_{2} \\
0 & S_{4}
\end{array}\right]
$$

expression (3.1) can be simplified to

$$
\left[\begin{array}{l}
x(T) \\
\lambda(T)
\end{array}\right]=\left[\begin{array}{c}
e^{A_{0} T} x(0) \\
e^{-A_{0}^{\prime} T} \lambda(0)
\end{array}\right]+\varepsilon\left[\begin{array}{c}
L_{1} x(0)+L_{2} \lambda(0)+e^{A_{0} T} S_{1} C-S_{1} C \\
L_{3} x(0)+L_{4} \lambda(0)
\end{array}\right] .
$$

Hence, adding boundary conditions from (2.12), we arrive to the following system of algebraic equations:

$$
\begin{gathered}
x(T)-\varepsilon L_{2} \lambda(0)=e^{A_{0} T} x(0)+\varepsilon\left(e^{A_{0} T} S_{1} C-S_{1} C+L_{1} x(0)\right), \\
\lambda(T)-\left(e^{-A_{0}^{\prime} T}+\varepsilon L_{4}\right) \lambda(0)=\varepsilon L_{3} x(0), \\
\lambda(T)-N x(T)=-N \bar{x}
\end{gathered}
$$

which in the matrix form can be written as

$$
\left[\begin{array}{ccc}
E & -\varepsilon L_{2} & 0 \\
0 & -e^{-A_{0}^{\prime} T}-\varepsilon L_{4} & E \\
N & 0 & -E
\end{array}\right]\left[\begin{array}{l}
x(T) \\
\lambda(0) \\
\lambda(T)
\end{array}\right]=\left[\begin{array}{c}
e^{A_{0} T} x_{0}+\varepsilon\left(e^{A_{0} T} S_{1} C-S_{1} C+L_{1} x_{0}\right) \\
\varepsilon L_{3} x_{0} \\
N \bar{x}
\end{array}\right]
$$


Multiplying (3.10) by $\left[\begin{array}{ccc}E & 0 & 0 \\ 0 & E & E \\ 0 & 0 & E\end{array}\right]$ on the left, we obtain

$$
\left[\begin{array}{ccc}
E & -\varepsilon L_{2} & 0 \\
N & -e^{-A_{0}^{\prime} T}-\varepsilon L_{4} & 0 \\
N & 0 & -E
\end{array}\right]\left[\begin{array}{c}
x(T) \\
\lambda(0) \\
\lambda(T)
\end{array}\right]=\left[\begin{array}{c}
e^{A_{0} T} x_{0}+\varepsilon\left(e^{A_{0} T} S_{1} C-S_{1} C+L_{1} x_{0}\right) \\
N \bar{x}+\varepsilon L_{3} x_{0} \\
N \bar{x}
\end{array}\right]
$$

Hence, if we denote the coefficient matrix in (3.11) by $M$, then it can be written as

$$
M=\left[\begin{array}{ccc}
E & -\varepsilon L_{2} & 0 \\
N & -e^{-A_{0}^{\prime} T}-\varepsilon L_{4} & 0 \\
N & 0 & -E
\end{array}\right]=\left[\begin{array}{cc}
K(\varepsilon) & 0 \\
K_{1} & -E
\end{array}\right]
$$

where

$$
K(\varepsilon)=\left[\begin{array}{cc}
E & -\varepsilon L_{2} \\
N & -e^{-A_{0}^{\prime} T}-\varepsilon L_{4}
\end{array}\right], \quad K_{1}=\left[\begin{array}{ll}
N & 0
\end{array}\right] .
$$

It is not difficult to show that

$$
\left[\begin{array}{cc}
K(\varepsilon) & 0 \\
K_{1} & -E
\end{array}\right]^{-1}=\left[\begin{array}{cc}
K^{-1}(\varepsilon) & 0 \\
K_{1} K^{-1}(\varepsilon) & -E
\end{array}\right]
$$

Hence, using the fact that

$$
K^{-1}(\varepsilon) \approx K^{-1}(0)-K^{-1}(0) \dot{K}(0) K^{-1}(0) \varepsilon
$$

we obtain the inverse matrix

$$
M^{-1} \approx\left[\begin{array}{ccc}
E+L_{2} e^{A_{0}^{\prime} T} N \varepsilon & -L_{2} e^{A_{0}^{\prime} T} \varepsilon & 0 \\
e^{A_{0}^{\prime} T} N-e^{A_{0}^{\prime} T} L_{4} e^{A_{0}^{\prime} T} N \varepsilon+e^{A_{0}^{\prime} T} N L_{2} e^{A_{0}^{\prime} T} N \varepsilon & e^{A_{0}^{\prime} T}-e^{A_{0}^{\prime} T} N L_{2} e^{A_{0}^{\prime} T} \varepsilon+e^{A_{0}^{\prime} T} L_{4} \varepsilon & 0 \\
N+N L_{2} e^{A_{0}^{\prime} T} N \varepsilon & -N L_{2} e^{A_{0}^{\prime} T} \varepsilon & -E
\end{array}\right]
$$

and, consequently, multiplying the both sides of (3.11) by $M^{-1}$ on the left, we obtain the following analytic formulae to determine values of $x(T), \lambda(0), \lambda(T)$ :

$$
\begin{aligned}
& {\left[\begin{array}{c}
x(T) \\
\lambda(0) \\
\lambda(T)
\end{array}\right]=\left[\begin{array}{c}
e^{A_{0} T} x_{0} \\
e^{A_{0}^{\prime} T} N e^{A_{0} T} x_{0}+e^{A_{0}^{\prime} T} N \bar{x} \\
N e^{A_{0} T} x_{0}-N \bar{x}
\end{array}\right]} \\
& +\varepsilon\left[\begin{array}{c}
e^{A_{0} T} S_{1} C-S_{1} C+L_{1} x_{0}+L_{2} e^{A_{0}^{\prime} T} N e^{A_{0} T} x_{0}-L_{2} e^{A_{0}^{\prime} T} N \bar{x} \\
e^{-A_{0}^{\prime} T} N e^{A_{0} T} S_{1} C-e^{A_{0}^{\prime} T} N S_{1} C+e^{A_{0}^{\prime} T} N L_{1} x_{0}+e^{A_{0}^{\prime} T} L_{3} x_{0} \\
-e^{A_{0}^{\prime} T} L_{4} e^{A_{0}^{\prime} T} N e^{A_{0} T} x_{0}+e^{A_{0}^{\prime} T} N L_{2} e^{A_{0}^{\prime} T} N e^{A_{0} T} x_{0} \\
-e^{A_{0}^{\prime} T} N L_{2} e^{A_{0}^{\prime} T} N \bar{x}+e^{A_{0}^{\prime} T} L_{4} e^{A_{0}^{\prime} T} N \bar{x} \\
N L_{2} e^{A_{0}^{\prime} T} N e^{A_{0} T} x_{0}-N L_{2} e^{A_{0}^{\prime} T} N \bar{x}+N L_{1} x_{0}+N e^{A_{0} T} S_{1} C-N S_{1} C
\end{array}\right] .
\end{aligned}
$$




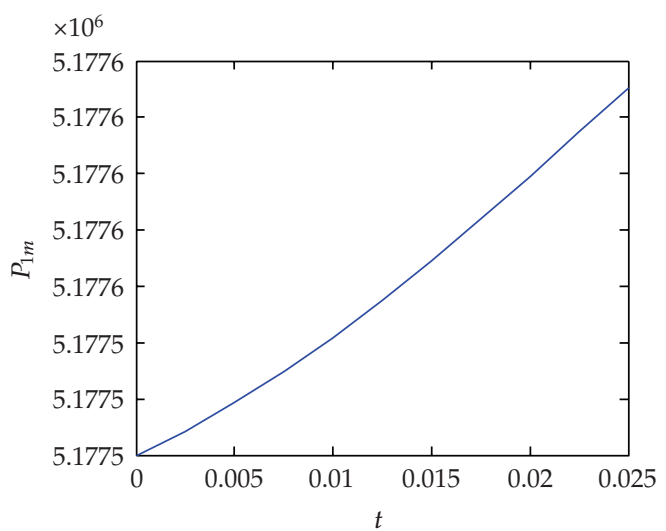

(a)

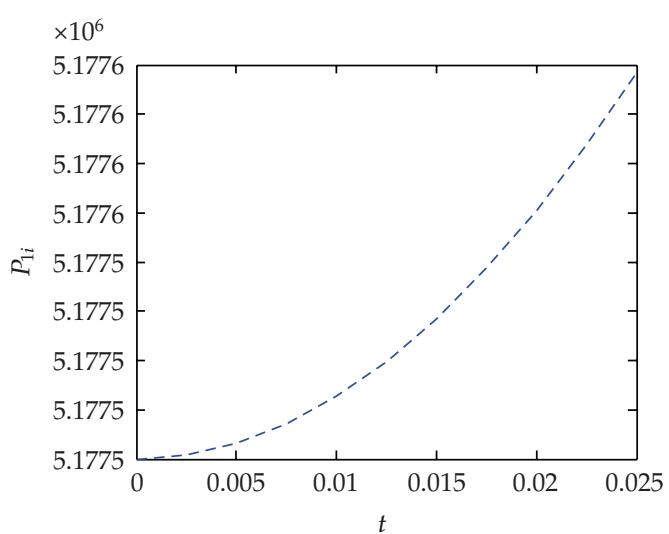

(b)

Figure 1: Dependence of $P_{1}$ on $t$.

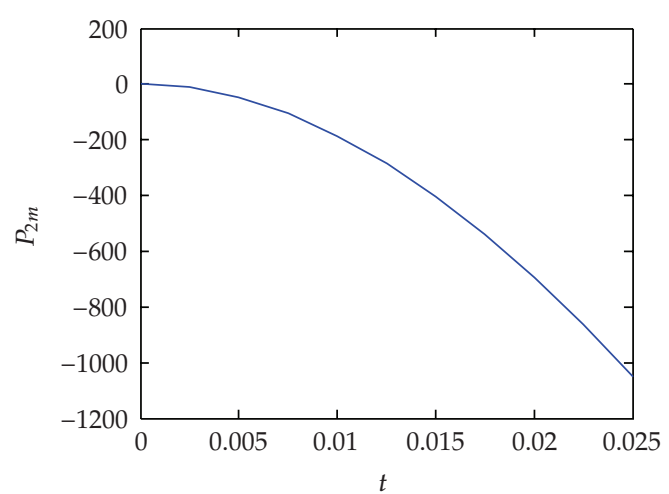

(a)

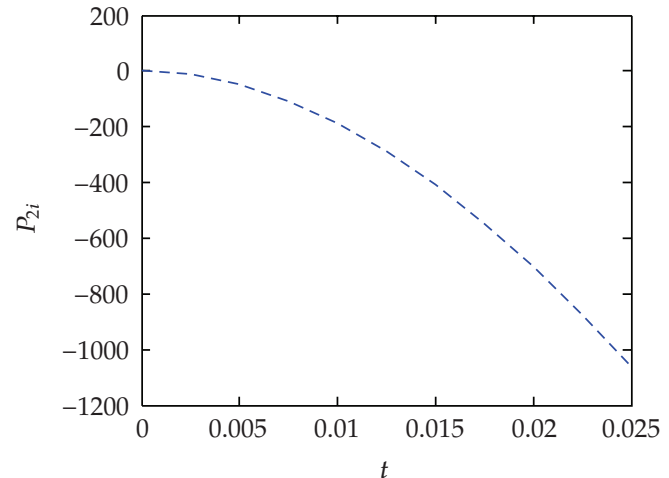

(b)

Figure 2: Dependence of $P_{2}$ on $t$.

Further, as in (3.1), using determined in (3.17) value $\lambda(0)$, it is possible to find $x\left(t_{i}\right), \lambda\left(t_{i}\right)$ in the form

$$
\begin{aligned}
{\left[\begin{array}{l}
x\left(t_{i}\right) \\
\lambda\left(t_{i}\right)
\end{array}\right]=} & e^{\left[\begin{array}{cc}
A_{0}+\varepsilon A_{1}-\varepsilon B \bar{R}^{-1} B^{\prime} \\
0 & -A_{0}^{\prime}-\varepsilon A_{1}^{\prime}
\end{array}\right] t_{i}}\left[\begin{array}{c}
x_{0} \\
\lambda(0)
\end{array}\right] \\
& +\left[e^{\left[\begin{array}{cc}
A_{0}+\varepsilon A_{1}-\varepsilon B \bar{R}^{-1} B^{\prime} \\
0 & -A_{0}^{\prime}-\varepsilon A_{1}^{\prime}
\end{array}\right]}-\left[\begin{array}{cc}
E & 0 \\
0 & E
\end{array}\right]\right]\left[\begin{array}{cc}
A_{0}+\varepsilon A_{1} & -\varepsilon B \bar{R}^{-1} B^{\prime} \\
0 & -A_{0}-\varepsilon A_{1}
\end{array}\right]^{-1}\left[\begin{array}{c}
C \varepsilon \\
0
\end{array}\right]
\end{aligned}
$$

for every $t_{i} \in\left[\begin{array}{ll}0 & T\end{array}\right]$. 


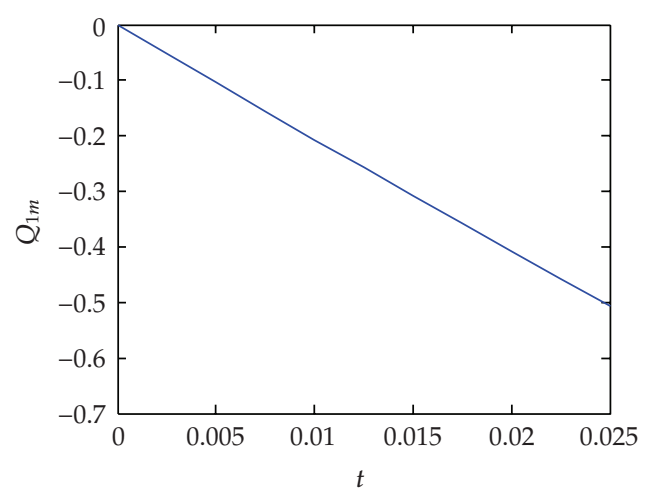

(a)

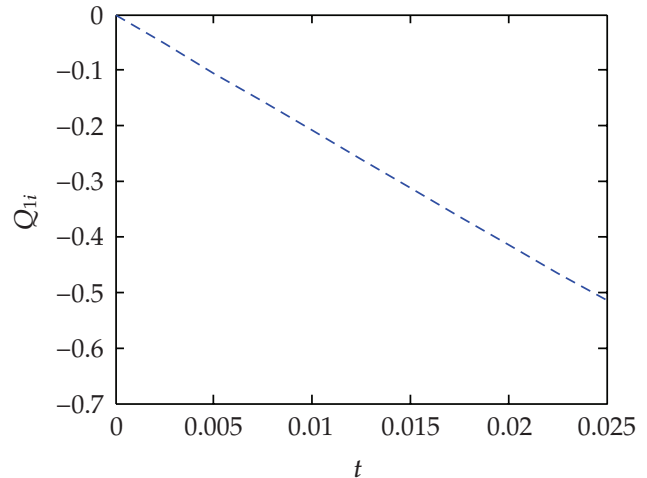

(b)

Figure 3: Dependence of $Q_{1}$ on $t$.

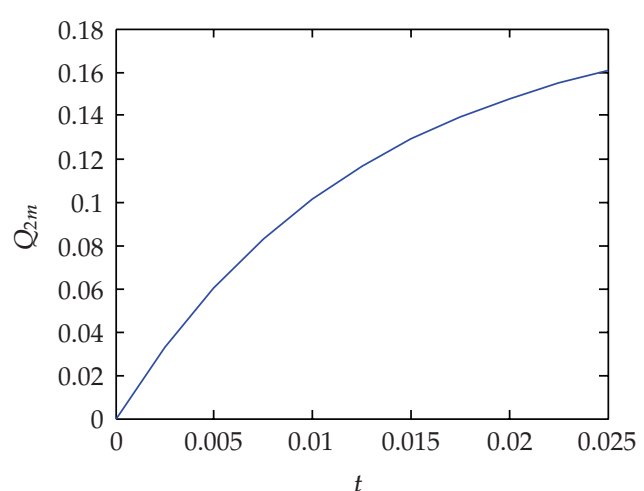

(a)

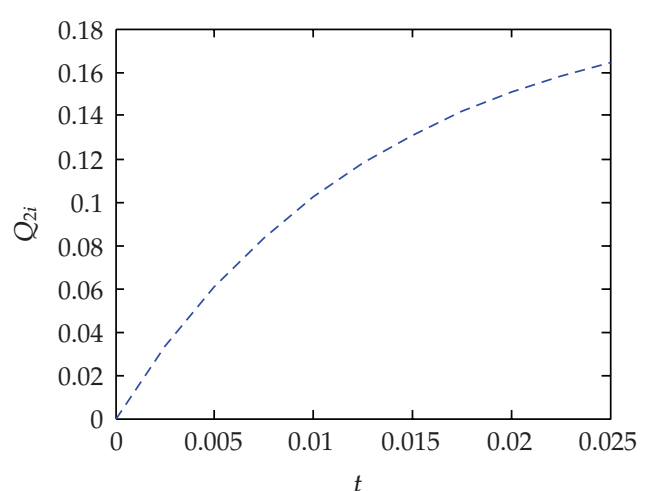

(b)

Figure 4: Dependence of $Q_{2}$ on $t$.

Therefore, expansions for $x\left(t_{i}\right)$ and $u\left(t_{i}\right)$ with respect to $\varepsilon$ can be obtained in the form

$$
\begin{aligned}
x\left(t_{i}\right)= & e^{A_{0} t_{i}}+\varepsilon\left(e^{A_{0} t_{i}} S_{1} C-S_{1} C+L_{11} x_{0}-L_{22} e^{A_{0}^{\prime} t_{i}} N e^{A_{0} t_{i}} x_{0}+L_{22} e^{A_{0}^{\prime} t_{i}} N \bar{x}\right), \\
\lambda\left(t_{i}\right)= & -N e^{A_{0} T} x_{0}+N \bar{x} \\
& +\varepsilon\left(N L_{22} e^{A_{0}^{\prime} t_{i}} N e^{A_{0} t_{i}} x_{0}-N L_{22} e^{A_{0}^{\prime} t_{i}} N \bar{x}-N L_{11} x_{0}-N e^{A_{0} t_{i}} S_{1} C-N S_{1} C\right), \\
u\left(t_{i}\right)= & -R^{-1} B^{\prime} \lambda\left(t_{i}\right) .
\end{aligned}
$$

Here $L_{11}, L_{22}$ are block matrices of matrix-solutions $L_{i}$ of Sylvester's equation $[12,13]$

$$
H_{1} L_{i}-L_{i} H_{1}=e^{H_{1} t_{i}} H_{2}-H_{2} e^{H_{1} t_{i}}
$$

for every $t_{i}$. 


\section{Computational Experiments}

On the basis of the obtained formulae, we have developed an algorithm and computer program using MATLAB system. In [5] an algorithm for solving problem (2.8)-(2.10) for the case when $C=0, N=2$ is developed. It is clear that for $N>2$ the algorithm will yield more accurate solutions. However, for computational comparison of the asymptotic method with the method described in [5], authors have considered only the case when $C=0, N=2$. It is possible to prove that in this case Sylvester's equation has infinitely many solutions. While using a special program in MATLAB one particular solution is determined.

In Figures 1,2,3, and 4 the graphs of the functions $P_{1}, Q_{1}, P_{2}$ and $Q_{2}$ for both algorithms are given. Namely, the graphs for the functions obtained using the algorithm introduced in $[5,14,15]$ are shown in solid lines and by the algorithm elaborated using the asymptotic method are given in dashed lines. A comparative analysis shows that the results for the functions $P_{1}, Q_{1}, P_{2}$, and $Q_{2}$ for both algorithms coincide with a sufficiently high accuracy.

\section{References}

[1] V. I. Shurov, Technology and Techniques of Oil Recovery, Nedra, Moscow, Russia, 1983.

[2] A. Kh. Mirzajanzadeh, I. M. Akhmetov, A. M. Khasaev, and V. I. Gusev, Technology and Techniques of Oil Recovery, A. Kh. Mirzajanzade, Ed., Nedra, Moscow, Russia, 1986.

[3] F. A. Aliev, M. A. Jamalbayov, and S. M. Nasibov, "Mathematical modeling of the well-bed system under the gaslift operation," TWMS Journal of Pure and Applied Mathematics, vol. 1, no. 1, pp. 5-13, 2010.

[4] F. A. Aliev, M. Kh. Ilyasov, and M. A. Jamalbekov, "Simulation of gas-lift well operation," Dokaldi of NAS of Azerbaijan, no. 4, pp. 30-41, 2008.

[5] F. A. Aliev, M. Kh. Ilyasov, and N. B. Nuriev, "Problems of mathematical simulation, optimization and control of gas lift," Dokaldi of NAS of Azerbaijan, no. 2, pp. 43-57, 2009.

[6] B. A. Bordyug, V. B. Larin, and A. G. Timashenko, Problems of Control of Walking Apparatus, Nukova Dumka, Kiev, Ukraine, 1985.

[7] X. Yuan, E. Caraballo, J. Little et al., "Feedback control design for subsonic cavity flows," Applied and Computational Mathematics, vol. 8, no. 1, pp. 70-91, 2009.

[8] R. Gabasov, E. M. Kirillova, and E. I. Poyasok, "Optimal real-time control of nondeterministic models on imperfect measurements of input and output signals," TWMS Journal of Pure and Applied Mathematics, vol. 1, no. 1, pp. 24-40, 2010.

[9] F. L. Chernousko, “Optimal ellipsoidal estimates of control and uncertain systems (survey)," Applied and Computational Mathematics, vol. 8, no. 2, pp. 135-151, 2009.

[10] F. A. Aliev and M. M. Mutallimov, "Algorithms for solving an optimal control problem with threepoint nonseparated boundary conditions," Problemy Upravleniya i Informatiki, no. 4, pp. 36-45, 2005.

[11] R. Gabasov, F. M. Kirillova, and N. S. Paulianok, "Optimal control of linear systems on quadratic performance index," Applied and Computational Mathematics, vol. 7, no. 1, pp. 4-20, 2008.

[12] R. Bellman, Introduction to Matrix Theory, Izdat. "Nauka", Moscow, Russia, 1969.

[13] F. A. Aliev and V. B. Larin, "About use of the Bass relations for solution of matrix equations," Applied and Computational Mathematics, vol. 8, no. 2, pp. 152-162, 2009.

[14] M. R. Soltanpour and M. Siahi, "Robust control of robot manipulator in task space," Applied and Computational Mathematics, vol. 8, no. 2, pp. 227-238, 2009.

[15] F. A. Aliev, A. N. Abbasov, and M. M. Mutallimov, "Algorithm for the solution of the problem optimization of the energy expenses at the exploitation of chinks by subsurface-pump installations," Applied Mathematics and Computation, vol. 3, no. 1, pp. 2-9, 2004. 


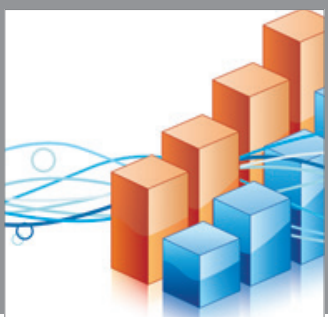

Advances in

Operations Research

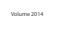

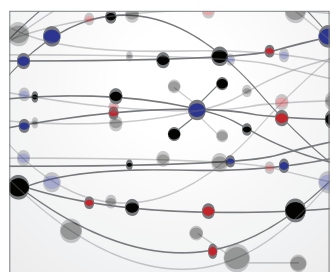

\section{The Scientific} World Journal
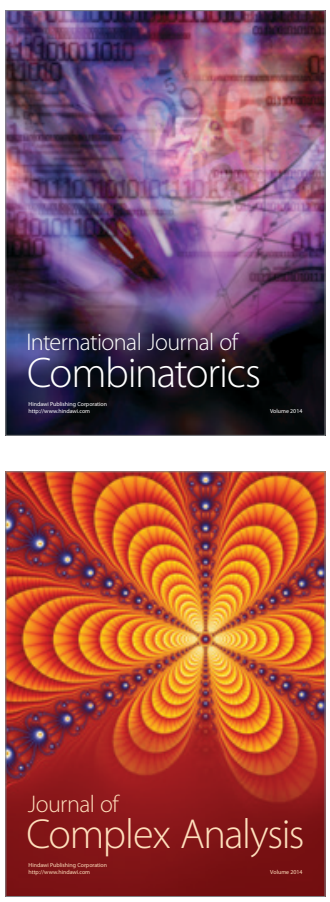

International Journal of

Mathematics and

Mathematical

Sciences
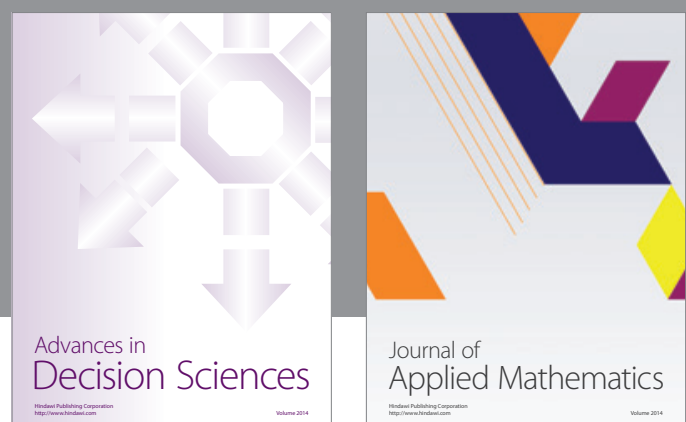

Journal of

Applied Mathematics
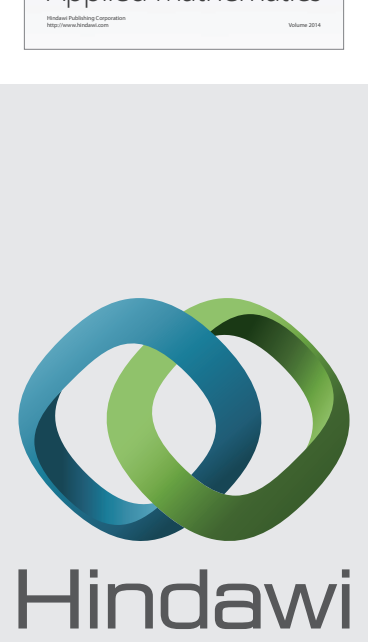

Submit your manuscripts at http://www.hindawi.com
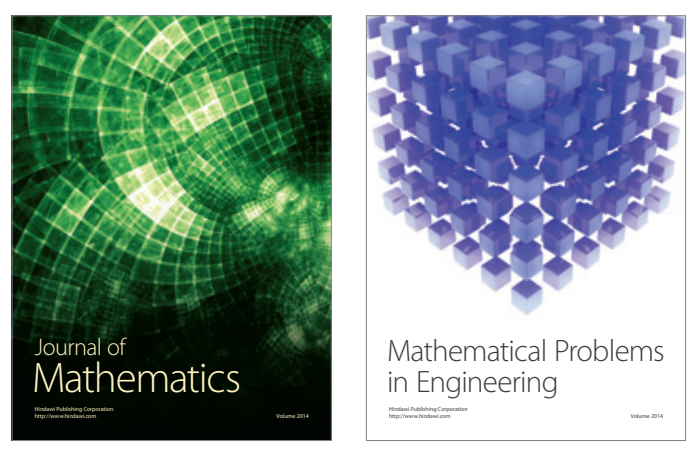

Mathematical Problems in Engineering
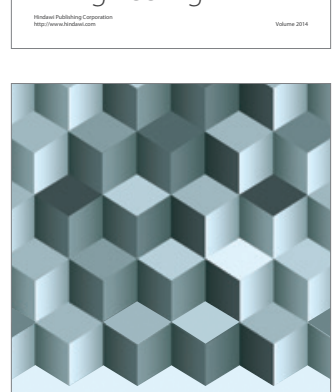

Journal of

Function Spaces
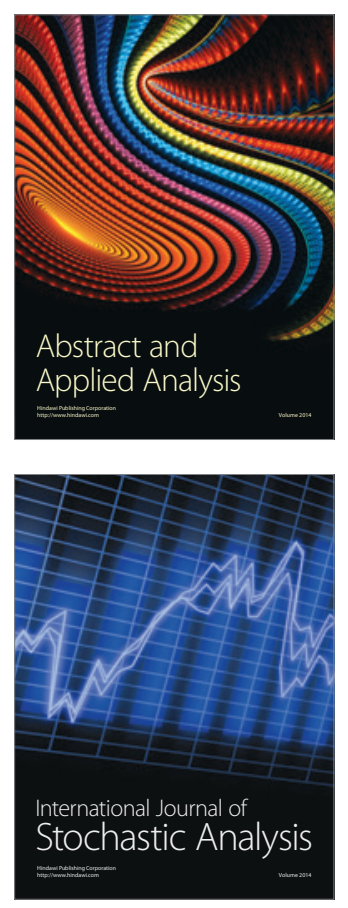

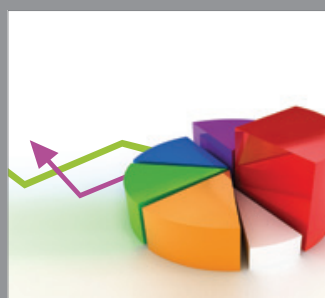

ournal of

Probability and Statistics

Promensencen
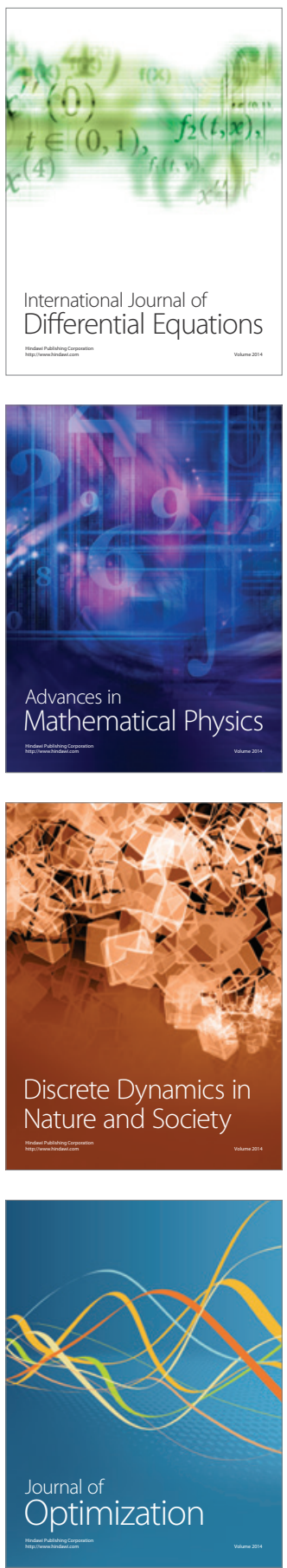